DOI: 10.2478/awutm-2014-0001 DE GRUYTER
OPEN
Analele Universităţii de Vest,

Timişoara

Seria Matematică - Informatică

LII, 1, (2014), 03- 16

\title{
Recurrent Inequalities and Some of their Applications to Picard and Mann Iterations
}

Vasile Berinde and Ştefan Măruşter

\begin{abstract}
Two lemmas concerning the superior bound of a numerical sequence satisfying a common recurrence inequality are given. As applications, the error estimations are obtained for the Picard and Mann iterations in the case of demicontractive mappings. Additional conditions that ensure the strong convergence in the same cases can be obtained on the basis of these lemmas.
\end{abstract}

AMS Subject Classification (2000). G.1.5

Keywords. Recurrent inequalities, Fixed points, Iterative procedures.

\section{Introduction}

Ostrowski (1967, [18]) was among the first authors to use a recurrent inequality for proving the T-stability of the Picard iteration of a contraction mapping in the framework of a general metric space. More precisely, Ostrowski's Lemma states that if the numerical sequence $\left\{a_{n}\right\}$ satisfies the recurrent inequality $a_{n+1} \leq \gamma a_{n}+\delta_{n}$ where $0<\gamma<1$ and $\delta_{n} \leq p$, then $\varlimsup \lim a_{n} \leq p /(1-m)$. As a corollary, he also obtains the convergence to zero of $\left\{a_{n}\right\}$ provided that $\delta_{n} \rightarrow 0$ as $n \rightarrow \infty$.

There exists a significant interest in developing such type of lemmas due to their applications in studying the strong convergence of various iteration processes for approximating fixed points (Picard, Mann, Halpern and Ishikawa 
iterations and numerous variations). In [5] collected most of these well known lemmas, while in $[6,7]$, detailed proofs of them are presented. Essentially, the technique of proof for such results is based on Cauchy's lemma, see for example [6], [7] and references therein. Often, the recurrent numerical inequalities are important by themselves, see for example in [2], where several generalizations of an Alber lemma [1] are given.

Below are presented three such lemmas. The choice to consider them here was rather casually but they are often used in studying the convergence properties of fixed point iterative schemes, while a third one refers to a recurrence inequality considered here.

Alber lemma (1979) [1].

Let $\left\{\lambda_{k}\right\}$ and $\left\{\gamma_{k}\right\}$ be sequences of nonnegative numbers and $\left\{\alpha_{k}\right\}$ be a sequence of positive numbers satisfying the conditions $\sum_{1}^{\infty} \alpha_{n}=\infty$, $\lim _{n \rightarrow \infty} \gamma_{n} / \alpha_{n} \rightarrow 0$.

Let the recursive inequality

$$
\lambda_{n+1} \leq \lambda_{n}-\alpha_{n} \psi\left(\lambda_{n}\right)+\gamma_{n}, n=1,2, \ldots,
$$

hold, where $\psi(\lambda)$ is a contiuous and nondecreasing function from $\mathbb{R}^{+}$to $\mathbb{R}^{+}$ such that it is positive on $\mathbb{R}^{+} \backslash\{0\}, \psi(0)=0, \lim _{t \rightarrow \infty} \psi(t)>0$. Then $\lambda_{n} \rightarrow 0$ as $n \rightarrow \infty$.

Tan-Xu lemma (1993) [22].

Suppose $\left\{a_{n}\right\}$ and $\left\{b_{n}\right\}$ are two sequences of nonnegative numbers such that $a_{n+1} \leq a_{n}+b_{n}$ for all $n \geq 1$. If $\sum_{n} b_{n}$ converges, then $\lim _{n} a_{n}$ exists.

A Lemma from [6] (2009).

Let $\left\{a_{n}\right\},\left\{b_{n}\right\}$ be sequences of nonnegative numbers and $0 \leq q<1$, so that

$$
a_{n+1} \leq q a_{n}+b_{n}, \text { for all } n \geq 0 \text {. }
$$

(1) If $\lim _{n \rightarrow \infty} b_{n}=0$, then $\lim _{n \rightarrow \infty} a_{n}=0$;

(2) If $\sum_{n=0}^{\infty} b_{n}<\infty$, then $\sum_{n=0}^{\infty} a_{n}<\infty$.

Note that (1.1) is the recurrent inequality considered by Ostrowski and that this Lemma completes the result of [18].

In this paper upper bounds of the sequence verifying the recurrence inequality (1.1) are done. Based on these bounds the convergence to zero of the generated sequence is proved. Some applications to Picard and Mann iterations are given for demicontractive mappings. 


\section{The upper bound in recurrence inequalities}

Lemma 2.1. Let $\left\{d_{n}\right\}$ be a nonnegative sequence satisfying

$$
d_{n+1} \leq a d_{n}+K \varepsilon_{n}, n=0,1, \ldots,
$$

where $0<a<1, K>0$ and $\left\{\varepsilon_{n}\right\}$ is a nonnegative sequence that satisfies the condition

$$
\frac{\varepsilon_{n+1}}{\varepsilon_{n}} \geq \mu>a, n=0,1, \ldots
$$

Then

$$
d_{n+1} \leq\left(\frac{a d_{0}}{\varepsilon_{0}}\left(\frac{a}{\mu}\right)^{n}+\frac{\mu K}{\mu-a}\right) \varepsilon_{n} \approx \frac{\mu K}{\mu-a} \varepsilon_{n}, n=0,1, \ldots
$$

Proof. Using the inequality (2.1), we obtain

$$
d_{n+1} \leq d_{0} a^{n+1}+K \sum_{i=0}^{n} \varepsilon_{i} a^{n-i} .
$$

Then it is simple to prove by induction on $n$ that

$$
\sum_{i=0}^{n} \varepsilon_{i} a^{n-i} \leq \frac{\mu}{\mu-a} \varepsilon_{n} .
$$

Taking into account that $\mu^{n} \varepsilon_{0} \leq \varepsilon_{n}$, we have

$$
\begin{aligned}
d_{n+1} & \leq d_{0} a^{n+1}+\frac{\mu K}{\mu-a} \varepsilon_{n} \leq \frac{a d_{0}}{\varepsilon_{0}}\left(\frac{a}{\mu}\right)^{n} \varepsilon_{n}+\frac{\mu K}{\mu-a} \varepsilon_{n} \\
& \leq\left(\frac{a d_{0}}{\varepsilon_{0}}\left(\frac{a}{\mu}\right)^{n}+\frac{\mu K}{\mu-a}\right) \varepsilon_{n} \approx \frac{\mu K}{\mu-a} \varepsilon_{n} . \square
\end{aligned}
$$

Remark 2.1. If $\varepsilon_{n} \rightarrow 0$, then condition (2.2) ensures a slow speed of convergence; however, it is, in some extent, reasonable because such a case often appears in the study of fixed point iterative schemes.

Lemma 2.2. Let $\left\{d_{n}\right\}$ be a numerical sequence satisfying the inequality (2.1) where $0<a<1, K>0$. Suppose the sequence $\left\{\varepsilon_{n}\right\}$ satisfies the conditions $0 \leq \varepsilon_{n} \leq 1, \forall n$ and

$$
\frac{\varepsilon_{n+1}}{\varepsilon_{n}} \geq e^{-\frac{1}{2}} \approx 0.607
$$

Then

$$
d_{n+1} \leq d_{0} a^{n+1}+\frac{2 K \varepsilon_{n}^{1-a}}{1-a} .
$$


Proof. As in the proof of Lemma 2.1 we have

$$
d_{n+1} \leq d_{0} a^{n+1}+K \sum_{i=0}^{n} \varepsilon_{i} a^{n-i} .
$$

We will prove by induction that

$$
\sum_{i=0}^{n} \varepsilon_{i} a^{n-i} \leq \frac{2 \varepsilon_{n}^{1-a}}{1-a} .
$$

The assertion is true for $n=0$ because $(2.5)$ becomes $\varepsilon_{0}<\left(\frac{2}{1-a}\right)^{\frac{1}{a}}$ and the function in the right hand side has the minimum value $14.561 \ldots$ Suppose (2.5) is true for $n \geq 0$. Using the value $n+1$ in (2.5) and the inductive hypothesis, we have

$$
\sum_{i=0}^{n+1} \varepsilon_{i} a^{n+1-i}=a \sum_{i=0}^{n} \varepsilon_{i} a^{n-i}+\varepsilon_{n+1} \leq \frac{2 a \varepsilon_{n}^{1-a}}{1-a}+\varepsilon_{n+1} .
$$

So we have to prove that

$$
2 a \varepsilon_{n}^{1-a}+(1-a) \varepsilon_{n+1} \leq 2 \varepsilon_{n+1}^{1-a},
$$

which can be proved in two stages:

$$
\text { (I) } 2 a \varepsilon_{n}^{1-a} \leq(1+a) \varepsilon_{n+1}^{1-a}, \quad(I I)(1-a) \varepsilon_{n+1} \leq(1-a) \varepsilon_{n+1}^{1-a} .
$$

The first inequality is equivalent to

$$
\frac{\varepsilon_{n+1}}{\varepsilon_{n}} \geq\left(\frac{2 a}{1+a}\right)^{\frac{1}{1-a}} \text {. }
$$

Let now $f:(0,1) \rightarrow \mathbb{R}$ be the function defined by $f(a)=\left(\frac{2 a}{1+a}\right)^{\frac{1}{1-a}}$. The first derivative of $f$ is positive, so the maximum value of this function is reached for $a=1$, but $\lim _{a \rightarrow 1} f(a)=e^{-1 / 2}$. Therefore the first inequality (I) is true for $n \geq 0$.

The second inequality (II) is also true because it is equivalent to $\varepsilon_{n+1} \leq 1$.

The key of the proofs of these lemmas are the inequalities (2.4) and (2.5) which give upper bounds for the sums appearing in their left hand side. Following the same procedures, we can obtain several (different) formulas for upper bound. For example, two improved estimators are

$$
\sum_{i=0}^{n} \varepsilon_{i} a^{n-i} \leq 2 a \varepsilon_{n-1}+\varepsilon_{n}
$$


and

$$
\sum_{i=0}^{n} \varepsilon_{i} a^{n-i} \leq \frac{2 a \varepsilon_{n}^{1-a}}{1-a} .
$$

The accuracy of the bounds given by $\left(2.4^{\prime}\right)$ is indeed remarkable, as it can be seen in Figure 1.

In this figure are drawn the functions $f(n):=\sum_{i=0}^{n} \varepsilon_{i} a^{n-1}$ and their upper bounds given by (2.4') for two cases of sequences $\left\{\varepsilon_{n}\right\}$ given by the functions $e(x)=0.8 x^{2} /\left(1+x^{3}\right)$ and $e(x)=\sin ^{2} x /(1+x)$, respectively. The graph of $f$ is drawn with bars, while the upper bounds are drawn with continuous lines.
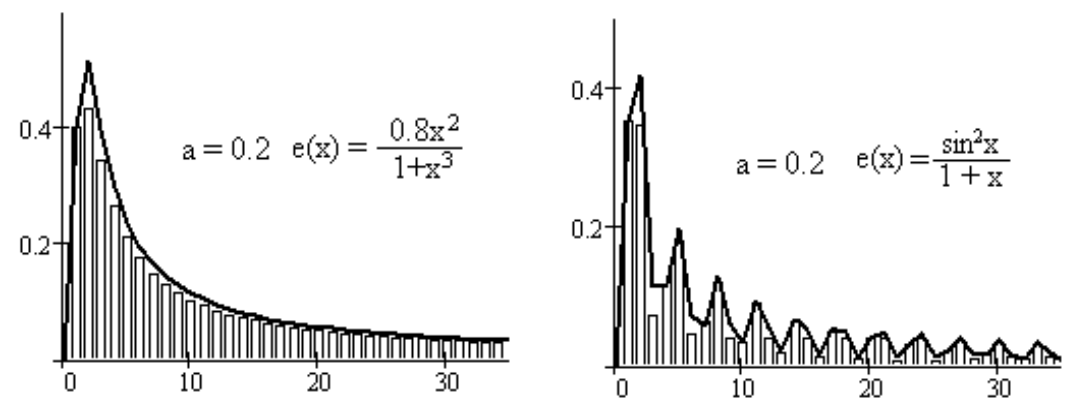

Figure 1: The accuracy given by the formula (2.4')

Remark 2.2. It must be pointed out that the upper bounds given by Lemma 2.2 have lower accuracy than those given by Lemma 2.1. But the fact that the inferior bound of $\varepsilon_{n+1} / \varepsilon_{n}$ does not depend of a can be a possible advantage of Lemma 2.2.

\section{Applications to Picard and Mann iterations for demi- contreactive mappings}

Recall that the concept of demicontractive mappings were defined in $[13,16$, 17], as follows.

Definition 3.1. Let $\mathcal{C}$ be a closed convex subset of a real Hilbert space $\mathcal{H}$ and let $T$ be a mapping from $\mathcal{C}$ into itself. Suppose that the set of fixed points Fix $(T)$ of $T$ is nonempty. The mapping $T$ is said to be demicontractive if

$$
\|T x-p\|^{2} \leq\|x-p\|^{2}+L\|x-T x\|^{2}, \forall x \in \mathcal{C}, p \in F i x(T),
$$


where $L>0$.

The demicontractivity and some smoothness conditions (for instance, the demi-closedness at zero) ensure the weak convergence of the Mann iteration, $x_{n+1}=\left(1-t_{n}\right) x_{n}+t_{n} T x_{n}$, where $\left\{t_{n}\right\}$ is the control sequence $[13,17]$. In [12] an example is given of a contraction (a contraction is more restrictive than a demicontractive mapping) defined on a bounded closed convex subset of a Hilbert space for which the Krasnoselski iteration (a particular case of the Mann iteration) does not converge. To get strong convergence, some additional conditions are needed.

The problem of additional conditions for strong convergence was discussed in several papers, including the old papers in which the concept of demicontractivity was introduced $[13,17]$. For example in [13] it is required, in addition, that $I-T$ maps closed bounded subsets of $C$ into closed subsets of $C$ (in particular, this is satisfied if $T$ is demicompact). In [17] the existence of a nonzero solution $h \in \mathcal{H}, h \neq 0$, of the variational inequality $\langle x-T x, h\rangle \leq 0, \forall x \in \mathcal{C}$ is required. It is obvious that the existence of a nonzero solution of this variational inequality occurs only in very particular cases; an example for linear equations is given in [17].

This subject is still being studied. In a relatively recent paper [8] it is required (as the main additional condition) that the mapping $T$ to be demicompact (Corollary 3.3). Note that this result was proved in [9] for a strictly pseudocontractive mapping (such mappings are more restrictive than demicontractive ones). The same type of additional conditions ( $T$ is demicompact or $\mathcal{C}$ is a compact subset of $\mathcal{H}$ ) appear in [14].

The problem of strong convergence is closely connected with the problem of error estimation (the estimation of $\left\|x_{n}-p\right\|$, where $\left\{x_{n}\right\}$ is a sequence approximating a fixed point $p$ ) and there exist several results for Mann iteration (or variants) and of some contractive type mappings. For example, in [11] is given the following error estimation of Mann iteration and for a montone, nonexpansive mapping:

$$
\left\|x_{n+1}-p\right\| \leq \frac{1}{\sqrt{n+1}}\left\|x_{1}-p\right\| .
$$

For a Lipschitzian strictly pseudocontractive mapping is given the estimation [15]:

$$
\left\|x_{n+1}-p\right\| \leq \rho^{n}\left\|x_{1}-p\right\|,
$$

where $\rho=1-k^{2} /\left[4\left(3+3 L+l^{2}\right)\right]$, and $L, k$ are the constants from Lipschitzian and strictly pseudocontractivity definitions. 
In the case of a Zamfirescu mapping $[3,5]$ the error estimate is given by the formula:

$$
\left\|x_{n+1}-p\right\| \leq \prod_{k=1}^{n}\left[1-\alpha_{k}(1-\delta)\right]\left\|x_{1}-p\right\|
$$

where $\left\{\alpha_{n}\right\}$ is control sequence and $\delta$ is the constant appearing in Zamfirescu type contraction.

More recent results on error estimation for Mann iteration have been reported in $[4,10,20]$.

The problem of error estimation was less approached for demicontractive mappings (there are no such results, to our best knowledge). The contribution of this paper is to give such estimates of the Mann iteration for the case of strong demicontractive mappings. Inequality (3.5), Theorem 3, provides an a posteriori error estimate. Based on this estimate, a new additional condition is obtained for strong convergence.

In what follow the demicontractivity will be strengthened in some extent: in place of (3.1) we consider the following condition

$$
\|T x-p\|^{2} \leq \alpha\|x-p\|^{2}+L\|x-T x\|^{2}, \forall(x, p) \in X \times F i x(T),
$$

where $\alpha \in(0,1)$ and $L \geq 0$. We will say that $T$ is strongly demicontractive. If $T$ is strongly demicontractive then the fixed point is unique. Indeed, if $q$ is another fixed point of $T$, then

$$
\|q-p\|^{2}=\left\|T q-\left.p\right|^{2} \leq \alpha\right\| q-p\left\|^{2}+L\right\| T q-q\left\|^{2}=\alpha\right\| q-p \|^{2},
$$

which implies $\alpha \geq 1$, contrary to the hypothesis.

Remark 3.1. The class of strictly demicontractive mappings coincides with the class of mappings having the (L,m)-property [19]. Taking into account that a mapping having $(L, m)$-property and a unique fixed point is actually a Kannan contraction [21], it results the coincidence between the strictly demicontractive mappings with fixed points and Kannan contractions.

Note that (3.2) is equivalent to

$$
\langle x-T x, x-p\rangle \geq \frac{1-\alpha}{2}\|x-p\|^{2}+\frac{1-L}{2}\|T x-x\|^{2}, \forall(x, p) \in C \times F i x(T) .
$$

But even this stronger demicontractivity condition does not ensure the convergence of Picard iteration. For example, the function $f:[-1,1] \rightarrow[-1,1]$ given by $f(x)=0.5\left(x^{3}-3 x\right)$ is strongly demicontractive for any pair $(\alpha, L)$ such that $0<\alpha<1, L>0$ and $0.16 \alpha+L>0.36$ (this relation was obtained by numerical search), but the Picard iteration does not converge. The 
Mann iteration also fails to converge if $0.8<t_{n}<1$ (for the above example). In both cases, the convergence of the process requires some additional specific conditions. It must be noted that in the case of Mann iteration such conditions may be imposed on the control sequence $\left\{t_{n}\right\}$.

\subsection{Picard iteration}

Theorem 3.1. Let $\mathcal{C}$ be a closed convex subset of a Hilbert space $\mathcal{H}$ and let $T$ be a mapping from $\mathcal{C}$ into itself. Assume that $T$ satisfies the following conditios:

(1) $\mathrm{T}$ is strictly demicontractive on $\mathrm{C}$;

(2) $\|T T x-T x\| \geq e^{-\frac{1}{4}}\|T x-x\| \approx 0.7788\|T x-x\|, \forall x \in \mathcal{C}$;

(3) $T$ is asymptotically regular at $x_{0}$, i.e. $\left\|T^{n+1} x_{0}-T^{n} x_{0}\right\| \rightarrow 0, n \rightarrow \infty$.

Then the Picard iteration starting at $x_{0}$ converges to $p$.

Proof. From strictly demicontractivity we obtain

$$
\left\|x_{n+1}-p\right\|^{2} \leq \alpha\left\|x_{n}-p\right\|^{2}+L\left\|x_{n+1}-x_{n}\right\|^{2}, n=0,1, \ldots
$$

Take $d_{n}=\left\|x_{n}-p\right\|^{2}, \varepsilon_{n}=\left\|x_{n+1}-x_{n}\right\|^{2}$ and so Lemma 2.2 can be applied. Note that, using condition (3), we can choose $n_{0}$ sufficiently large so that $\left\|T^{n+1} x_{0}-T^{n} x_{0}\right\| \leq 1$ for $n \geq n_{0}$. So $\varepsilon_{n} \leq 1$ and all conditions of Lemma 2.2 are satisfied.

Remark 3.2. (on condition (2) in Theorem 3.1)

If $T$ is a real function, $f:[a, b] \rightarrow[a, b]$, differentiable on $[a, b]$, then, using the Mean Value Theorem, we get

$$
\frac{\|f(f(x))-f(x)\|}{\|f(x)-x\|}=f^{\prime}(\xi)
$$

where $\xi=(1-\eta) x+\eta f(x) \in[a, b], \eta \in(0,1)$. Thus condition (2) becomes $f^{\prime}(x) \geq e^{-\frac{1}{2}}, \forall x \in[a, b]$.

The example below presents a real function which satisfies the conditions of Theorem 3.1.

Example 3.1. Let $C=[-0.5,0.5]$ and let $f$ be a function $f:[-0.5,0.5] \rightarrow$ $[-0.5,0.5]$ defined by

$$
f(x)= \begin{cases}-x+0.8 x^{3} & \text { if } x<0 \\ -x & \text { if } x \geq 0 .\end{cases}
$$

This function is strictly demicontractive with $\alpha=0.1, L=0.32$, and it satisfies $|f(f(x))-f(x)| /|f(x)-x| \geq 0.888 \ldots, \forall x \in[-0.5,0.5]$. Note that $f$ has the unique fixed point $p=0$ and that it is not a contraction on $[-0.5,0.5]$. 


\subsection{Mann iteration}

To simplify the exposition, we consider the Mann iteration with a constant control sequence, $t_{n}=t, n=0,1, \ldots$ (usually such a Mann iteration is referred as Krasnoselski iteration, see [5]). The general case together with the condition $0<a \leq t_{n} \leq b<1$ is quite similar to this particular case.

Theorem 3.2. Let $\mathcal{C}$ be a closed convex subset of a Hilbert space $\mathcal{H}$ and let $T$ be a mapping from $\mathcal{C}$ into itself. Assume that $T$ is strictly demicontractive on $\mathcal{C}$ with $0<L<1$. Then the Mann iteration with $0<t<1-L$ converges to $p$.

Proof. Using (3.3) it obtains

$$
\left\|x_{n+1}-p\right\|^{2} \leq[1-t(1-\alpha)]\left\|x_{n}-p\right\|^{2}-\left[t(1-L)-t^{2}\right]\left\|x_{n}-T x_{n}\right\|^{2} .
$$

Because $t<1-L$ we have $t(1-L)-t^{2}>0$ and because, obviously, $0<$ $\delta:=1-t(1-\alpha)<1$ we obtain $\left\|x_{n+1}-p\right\|^{2} \leq \delta\left\|x_{n}-p\right\|^{2}$.

Theorem 3.3. Let $T$ be a mapping defined on the Hilbert space $\mathcal{H}$ with values in $\mathcal{H}$ and assume that $T$ satisfies the following conditios:

(1) $T$ is strictly demicontractive on $\mathcal{H}$ with $0<\alpha<1, L>1$;

(2) There exist $\eta>\sqrt{a}$ such that $\|T y-y\| \geq \eta\|T x-x\|, \forall x \in \mathcal{H}$ and $t$ satisfying $\frac{1-\eta^{2}}{1-\alpha}<t \leq \frac{1}{1-\alpha}$, where $a=1-t(1-\alpha)$ and $y=x+t(T x-x)$;

(3) $T$ is asymptotic regular at $x_{0}$, i.e. $\left\|T^{n+1} x_{0}-T^{n} x_{0}\right\| \rightarrow 0, n \rightarrow \infty$.

Then the Mann iteration with constant control sequence, $t_{n}=t$, starting at $x_{0}$ converges to $p$ and a posteriori error is given by

$$
\begin{gathered}
\left\|x_{n+1}-p\right\| \leq\left\|x_{n+1}-x_{n}\right\| \sqrt{\frac{a\left\|x_{0}-p\right\|^{2}}{\left\|x_{1}-x_{0}\right\|^{2}}\left(\frac{a}{\eta^{2}}\right)^{n}+\frac{\eta^{2} K}{\eta^{2}-a}} \approx \\
\sqrt{\frac{\eta^{2} K}{\eta^{2}-a}}\left\|x_{n+1}-x_{n}\right\|,
\end{gathered}
$$

where $K=t^{-1}(t-1+L)$.

Proof. Using the obvious equality $T x_{n}-x_{n}=t^{-1}\left(x_{n+1}-x_{n}\right)$ and (3.4) we have

$$
\left\|x_{n+1}-p\right\|^{2} \leq[1-t(1-\alpha)]\left\|x_{n}-p\right\|^{2}+t^{-1}(t-1+L)\left\|x_{n+1}-x_{n}\right\|^{2} \text {. }
$$


We can now apply Lemma 2.1 with $d_{n}=\left\|x_{n}-p\right\|^{2}, \varepsilon_{n}=\left\|x_{n+1}-x_{n}\right\|^{2}$ and $a, K$ given above. The condition $\varepsilon_{n+1} / \varepsilon_{n} \geq \mu>a$ required by this lemma can be proved from conditon (2)and from $\frac{1-\eta^{2}}{1-\alpha}<t$. Indeed, we have

$$
\begin{aligned}
\frac{\varepsilon_{n+1}}{\varepsilon_{n}} & =\frac{\left\|x_{n+2}-x_{n+1}\right\|^{2}}{\left\|x_{n+1}-x_{n}\right\|^{2}}=\frac{\left\|T x_{n+1}-x_{n+1}\right\|^{2}}{\left\|T x_{n}-x_{n}\right\|^{2}} \\
& \geq \eta^{2}>1-t(1-\alpha)=a,
\end{aligned}
$$

that is the condition of Lemma 2.1 is satisfied with $\mu=\eta^{2}$. Now (3.5) follows from (2.3).

Remark 3.3. The condition (2) allows $t=1 /(1-a)$, that is a value greater than 1. Usually the control sequence of Mann iteration belongs to $(0,1)$, ensuring thus the preservation of the generated sequence in $C$. However there exist some particular iterative schemes of Mann type for which the control sequence does not belog to $(0,1)$ and that still the generated sequence converges to some fixed point (in this case $C=\mathcal{H}$ or the belongingness of the sequence to $C$ is a condition). A typical case is the projection method for solving convex feasibility problem.

\subsection{The linear case in a finite dimensional space}

Suppose $T: \mathbb{R}^{m} \rightarrow \mathbb{R}^{m}$ is a linear mapping, $T x=A x-b$, and $p$ is its fixed point. The following equalities are obvious:

$$
\begin{aligned}
& T x-x=(A-I)(x-p) \\
& T x-p=A(x-p) \\
& T y-y=(A-I)[I+t(A-I)](x-p),
\end{aligned}
$$

where $y=(1-t)+t T x$ and $I$ is the unitary matrix.

The first two requirements of Theorem 3 are satisfied in this case, provided that some conditions are fulfilled.

The condition (1).

Suppose that there exists $(A-I)^{-1}$. If $\alpha \in \mathbb{R}$ is any number such that $0<\alpha<1$, we can chose $L>0$ such that

$$
\|A\|^{2} \leq \alpha+L\left\|(A-I)^{-1}\right\|^{-2} .
$$

From this we have

$$
\|A\|^{2}\|x-p\|^{2} \leq \alpha\|x-p\|^{2}+L\left\|(A-I)^{-1}\right\|^{-2}\|x-p\|^{2},
$$

and the strongly demicontractivity condition is obvious.

The condition (2). 
Suppose $A$ satisfies the following two conditions:

(a) $A-I$ is invertible;

(b) The function $g(t)=\left\|[I+t(A-I)]^{-1}(A-I)^{-1}\right\|$ is well defined for $0<t \leq \frac{1}{1-\alpha}$ and

$$
\max _{0 \leq t \leq 1 /(1-\alpha)} g(t)=M<\infty
$$

If we define $\eta$ as $\eta=\frac{1}{M\|A-I\|}$ we have

$$
\left\|[(A-I)(I+t(A-I))]^{-1}\right\|^{-1} \geq \eta\|A-I\|,
$$

and

$$
\begin{aligned}
\|T y-y\| & =\|(A-I)[I+t(A-I)](x-p)\| \\
& \geq\left\|[(A-I)(I+t(A-I))]^{-1}\right\|^{-1}\|x-p\| \\
& \geq \eta\|A-I\|\|x-p\| \geq \eta\|(A-I)(x-p)\|=\eta\|T x-x\|,
\end{aligned}
$$

i.e. the condition (2) is satisfied.

Remark 3.4. The conditions (a) and (b) seem to be relatively strong; however there exist matrices with diagonal dominance which satisfy the both conditions. For example, the matrix

$$
A=\left(\begin{array}{llll}
0.2 & 0.1- & 0.4 & 0.1 \\
0.3 & 0.2- & 0.1 & 0.1 \\
0.0- & 0.2 & 0.5 & 0.0 \\
0.1 & 0.2 & 0.1 & 0.3
\end{array}\right)
$$

satisfy both (a) and (b). Indeed, a direct computation shows that $A-I$ is invertible and the linear function $T x=A x+b, b=(-0.1,-0.6,-1,0.8)^{T}$, is strongly demicontractive, the best constants being $\alpha=0.208, L=3.092$. The function $g$ is increasing on $[0,1 /(1-\alpha)]$, so $M=g(1 /(1-\alpha)$. The other numerical constants are $\eta=0.064, \theta=2.042 \times 10^{-3}, t \in[1.26,1.263]$ (in our experiment $t=1.26), a=2.08 \times 106^{-3}$.

\section{Some examples}

The accuracy of the error estimation (3.5) depends in some extent of the constant $L$ appearing in the condition of strong demicontractivity. The following examples show the accuracy of the proposed estimation in the case of 
a strictly demicontractive real function and of a linear function from $\mathbb{R}^{4}$ to $\mathbb{R}^{4}$.

The first example is a function $f:[0.5,1.5] \rightarrow[0.5,1.5]$, given by $f(x)=$ $0.05 x^{2}+0.8 x+0.15$. This function has the unique fixed point $p=1$ and it is strictly demicontractive on $[0.5,1.5]$ with the constants $\alpha=0.35, L=90$. The condition (2) of Theorem 3 is also satisfied and $\eta=0.777 \ldots$ The value of $t$ can be chosen between $\left(1-\eta^{2}\right) /(1-\alpha)=0.61,1 /(1-\alpha)=1.538$ (in our numerical example $t=1.538)$. So, the conditions (1) and (2) of Theorem 3.1 are satisfied.

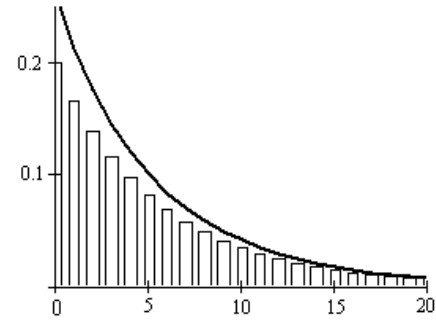

(a)

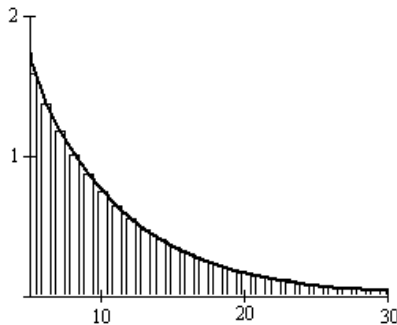

(b)

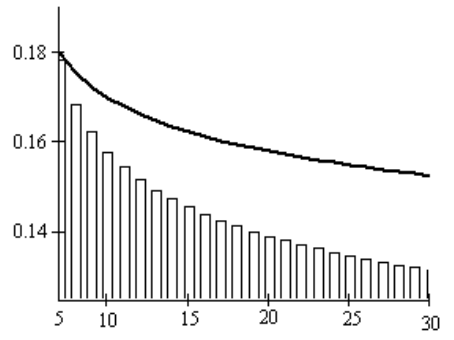

(c)

Figure 2: The accuracy given by estimation (3.5) the graphs (a) and (b) and by $(\mathrm{Z})$ the graph $(\mathrm{c})$.

In the Figure 2 (a) are depicted the true errors of the sequence $\left\{x_{n}\right\}$, that is the values $\left|x_{n}-p\right|, n=10, \ldots, 40$ (with bars), and the error estimations, that is the values $\sqrt{\eta^{2} K /\left(\eta^{2}-a\right)}\left|x_{n+1}-x_{n}\right|, n=10, \ldots, 40$ (with continuous lines).

The second example is a mapping $T: \mathbb{R}^{4} \rightarrow \mathbb{R}^{4}$ given by $T x=A x+b$, where $A$ and $b$ are given above (Remark 6$)$. The error estimation accuracy can be seen in the Figure 2 (b) (true errors with bars and estimation with a continuous line)

In the Figure 2 (c) are presented the true error and estimation error of Mann iteration given by formula $(\mathrm{Z})$ with control sequence $t_{n}=1 / n$ for the real function considered in the first example. Note that the function $f$ from this example verifies the condition $|f(x)-f(y)| \leq \delta|x-y|+2 \delta|x-f(x)|$ for $\delta \geq 0.93$, which is a consequence of Zamfirescu's contraction condition. 


\section{Acknowledgements}

The first authors' research was supported by the Grants PN-II-RU-TE-20113-239 and PN-II-ID-PCE-2011-3-0087 of the Romanian Ministry of Education and Research.

\section{References}

[1] Ya.I.Alber, On the solution of equations and variational inequalities with maximal monotone operators, Soviet mathematics Doklady, 20, (1979), 871-876

[2] Ya.I.Alber, C.E.Chidume, and H.Zegeye, Approximationg fixed points of total asymptotically nonexpansive mappings, Fixed Point Theory and Appl., 2006, Article ID 10673

[3] G.V.R.Babu and K.N.V.V.Vara Prasad, Mann iteration converges faster than Ishikawa iteration for the class of Zamfirescu operators, Fixed Point Theory and Applications, (2006), Article ID 49615

[4] V.Berinde, Approximating fixed points of weak contractions using the Picard iterations, Nonlinear Analysis Forum, (2004)

[5] V.Berinde, Iterative approximation of fixed points, Lecture Notes in Mathematics, (2007)

[6] V.Berinde, On a family of first order difference inequalities used in the iterative approximation of fixed points, Creative Math. and Inf., 18, (2009), 110-122

[7] V.Berinde, A note on a difference inequality used in the iterative approximation of fixed points, Creative Math. and Inf., 18, (2009), 6-9

[8] D.Boonchari and S.Saejung, Construction of common fixed points of a countable family of $\lambda$-demicontractive mappings in arbitrary Banach spaces, Appl. Math. Comput., 216, (2010), 173-178

[9] C.E.Chidume, M.Abbas, and B.Ali, Convergence of the Mann iteration algorithm for a class of pseudocontractive mappings, Appl. Math. Comput., 194, (2007), $1-6$

[10] L.Ciric, A.Rafiq, S.Radenovic, M. Rajovic, and J.S.Ume, On Mann implicit iteration for strongly accretive and strongly pseudo-contractive mappings, Appl. Math. Comput., 198, (2008), 128-137

[11] W.G.Dotson, An iterative process for nonlinear monotonic nonexpasive operators in Hilbert space, Mathematics of Computation, 32, (1978), 223-225

[12] A.Genel and J.Lindenstrauss, An example concerning fixed points, Israel J. Mathematics, 22, (1975), 81-86

[13] T.L.Hicks and J.D.Kubicek, On the Mann iteration process in a Hilbert spaces, J. Math. Anal. Appl., 59, (1977), 489-504 
[14] S.M.Kang, A.Rafiq, and N.Hussain, Weak and strong convergence of fixed points of demicontractive mappings in smooth Banach spaces, Int. J. Pure Appl. Math., 84, (2013), 251-268

[15] Liwei Liu, Proc. Amer. Math. Soc., 125, (1997), 1363-1366

[16] St.Maruster, Sur le calcul des zeros d'un operateur discontinu par iteration, Canad. Math. Bull., 16, (1973), 541-544

[17] St.Maruster, The solution by iteration of nonlinear equations in Hilbert spaces, Proc. Amer. Math.Soc., 63, (1977), 69-73

[18] A.M.Ostrowski, The round-of stability of iterations, Zeitschrift für angewandte mathematik und mechanik, 47, (1967), 77-81

[19] Y.Qing and B.E.Rhoades, T-stability of Picard iteration in metric spaces, Fixed Point Theory and Applications, 2008, Article ID418971

[20] D.R.Sahu and A.Petrusel, Strong convergence of iterative methods by strictly pseudocontractive mappings in Banach spaces, Nonlinear Analysis, 74, (2011), 60126023

[21] M.De la Sen, Some combined relations between Contractive mappings, Kannan mappings, Reasonable Expansive mappings, and T-stability, Fixed Point Theory and Applications, (2009), Article ID815637

[22] K.K.Tan and H.K.Xu, Approximating fixed points of nonexpansive mappings by the Ishikawa iteration process, J. Math. Anal. Appl., 178, (1993), 301-308

Vasile Berinde

North University of Baia Mare, Romania

E-mail: vasile_berinde@yahoo.com

Ştefan Măruşter

West University of Timişoara, Romania

E-mail: maruster@info.uvt.ro

Received: 16.06.2014

Accepted: 30.07.2014 